\title{
Rapid Progressive Peripheral Ulcerative Keratitis Associated with Longstanding Rheumatoid Arthritis
}

WON-SEOK LEE, MD, PhD, Division of Rheumatology, Department of Internal Medicine, Research Institute of Clinical Medicine of Chonbuk National University-Biomedical Research Institute of Chonbuk National University Hospital; YUN-JUNG CHOI, MD, Division of Rheumatology, Department of Internal Medicine, Research Institute of Clinical Medicine of Chonbuk National University-Biomedical Research Institute of Chonbuk National University Hospital; WAN-HEE YOO, MD, PhD, Division of Rheumatology, Department of Internal Medicine, Research Institute of Clinical Medicine of Chonbuk National University-Biomedical Research Institute of Chonbuk National University Hospital, Jeonju, South Korea. Address correspondence to Dr. W.H. Yoo, Division of Rheumatology, Department of Internal Medicine, Research Institute of Clinical Medicine of Chonbuk National University-Biomedical Research Institute of Chonbuk National University Hospital, San 2-20 Geumam-dong, Deokjin-gu, Jeonju, Jeonbuk, 561-180, South Korea.

E-mail: ywhim@jbnu.ac.kr. J Rheumatol 2016;43:1909-10; doi:10.3899/jrheum.160262

Peripheral ulcerative keratitis (PUK) is a unilateral crescent-shaped stromal inflammation that has long been associated with collagen vascular disease such as rheumatoid arthritis (RA) ${ }^{1}$. PUK in RA generally appears late in the disease course, and usually signals worsening of the systemic disease ${ }^{2,3}$. Tumor necrosis factor- $\alpha$ inhibitor (TNFi) has been reported to be effective in RA-associated PUK cases refractory to conventional immunomodulatory therapy 4,5 .

A 58-year-old woman with a 35-year history of RA (Figure 1) presented to our clinic with ocular pain and redness. Her symptoms had occurred 3 weeks before, and she had been treated with eyedrops at a local clinic. Since 2006, she had been treated with etanercept $(50 \mathrm{mg} /$ week $)$ and leflunomide (10 mg/day) for RA. Ocular examination revealed circumferential PUK (Figure 2A). The patient was initially treated with high-dose prednisolone $(1 \mathrm{mg} / \mathrm{kg})$; however, her condition worsened within 1 week (Figure 2B). Although previously treated with TNFi, her RA symptoms and PUK worsened, after which treatment was altered to tocilizumab (162 mg biweekly). After 3 weeks, corneal melting was rapidly progressive (Figure $2 \mathrm{C}$ ). However, she refused further surgical treatment.

Despite improvements in new immunomodulatory therapy, the outcome of PUK depends on the accompanying disease, as well as timely diagnosis and treatment ${ }^{2}$. The authors suggest that this patient's refractory response to treatment was due to the progression of symptoms prior to starting aggressive treatment. This image highlights the importance of close communication between ophthalmologists and rheumatologists for early diagnosis of PUK.

\section{REFERENCES}

1. Mondino BJ. Inflammatory diseases of the peripheral cornea. Ophthalmology 1988;95:463-72.

2. Yagci A. Update on peripheral ulcerative keratitis. Clin Ophthalmol 2012;6:747-54.

3. Foster CS, Forstot SL, Wilson LA. Mortality rate in rheumatoid arthritis patients developing necrotizing scleritis or peripheral ulcerative keratitis. Effects of systemic immunosuppression. Ophthalmology 1984;91:1253-63.

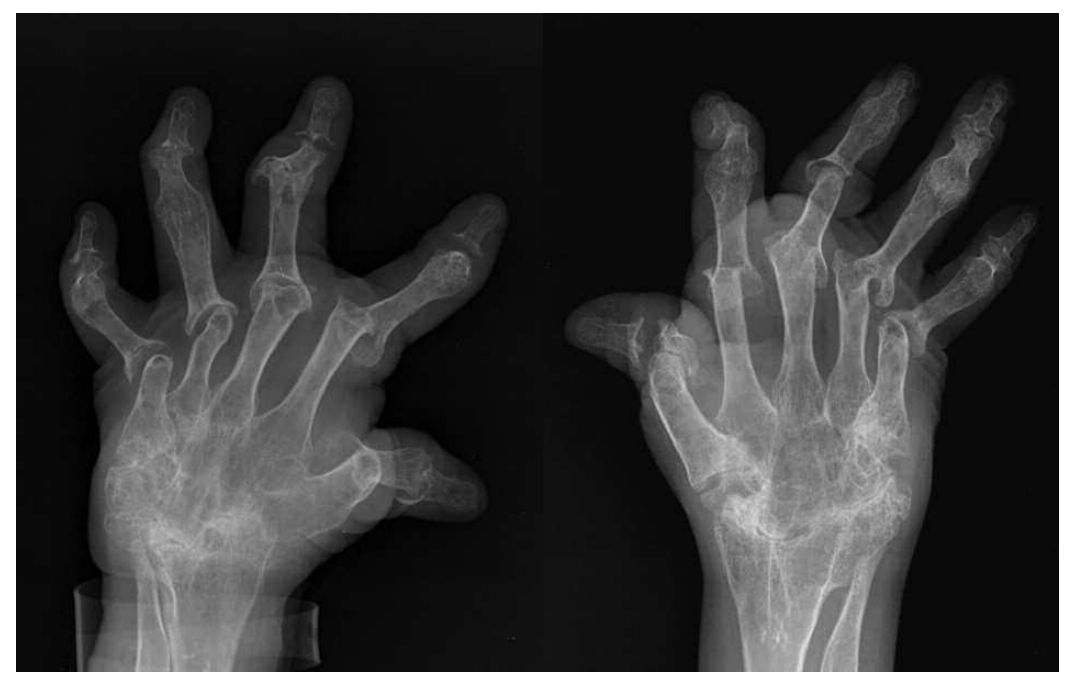

Figure 1. Radiographs of both hands showing severe rheumatoid arthritis affecting both wrists and hands. The deformity limits the movement of multiple joints in both hands. 


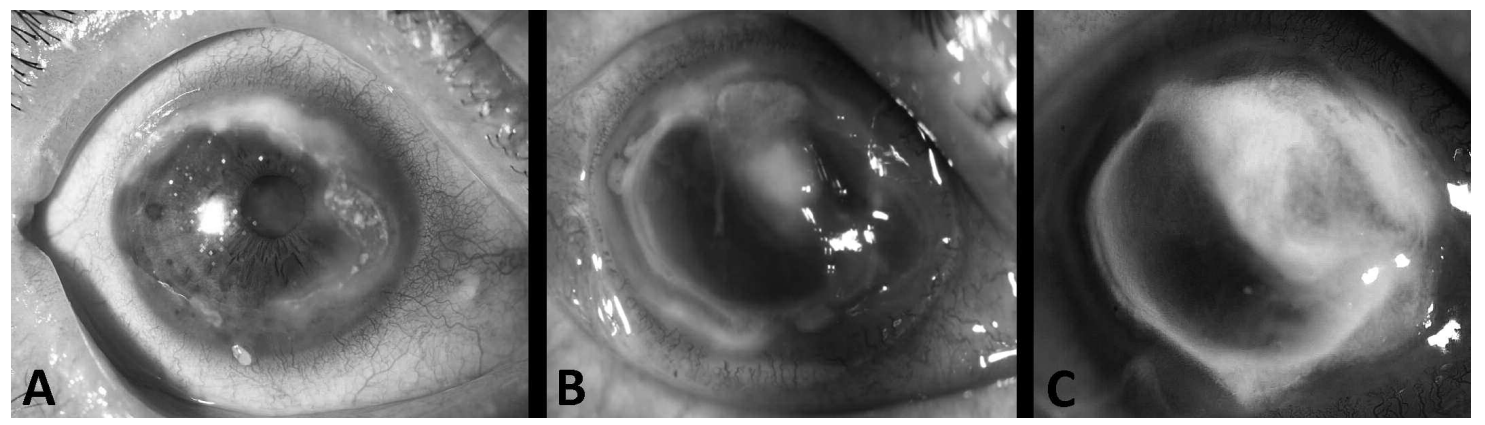

Figure 2. (A) Slit lamp photograph of a patient with rheumatoid arthritis demonstrates peripheral corneal stromal thinning and overlying epithelial defect. (B) After 1 week, the corneal ulceration had rapidly progressed. (C) After 3 weeks, slit lamp photograph showing severe corneal scarring as a consequence of peripheral ulcerative keratitis.

4. Atchia II, Kidd CE, Bell RW. Rheumatoid arthritis-associated necrotizing scleritis and peripheral ulcerative keratitis treated successfully with infliximab. J Clin Rheumatol 2006;12:291-3.
5. Antao SF, Ayoub T, Tahir H, Parmar DN. Stabilization of bilateral progressive rheumatoid corneal melt with infliximab. Case Rep Ophthalmol Med 2012;2012:173793. 